\title{
Rheumatoid Arthritis and Tuberculosis Link, 3 Cases of T.B. Tenosynovitis Wrist
}

\author{
Safa Eldin Abaza* and Sam Nashi \\ Consultant Orthopedic Surgeon, Gulf Diagnostic Center Hospital, UAE
}

*Corresponding author: Safaa Eldin Abaza, Consultant Orthopedic Surgeon, Gulf Diagnostic Center Hospital- Abu Dhabi, UAE.

Received Date: August 23, 2018

Published Date: September 17, 2018

\begin{abstract}
Three cases of sever synovitis and tenosynovitis of the wrist were sent to the author for surgical synovectomy and tenosynovectomies after failure of the medical treatment with biological and disease modifying drugs all of them turn to be tuberculous tenosynovitis after incidental soft tissue and synovial fluid culture, a discussion for the link between both different entity diseases and Awareness of Drug-specific risk of tuberculosis in patients with rheumatoid arthritis.
\end{abstract}

\section{Introduction}

Rheumatoid arthritis is best characterized as an immune mediated inflammatory disease (IMID). Within a framework that recognizes both immunological activation and inflammatory pathways is caused by a misfiring immune system (autoimmunity) attacking the healthy cells in the joints, causing inflammation, pain, swelling, stiffness and loss of function in the joints. It can affect any joint but is common in the wrist and fingers.

In a Comparative study of the synovial histology in rheumatoid arthritis, spondyloarthropathy, and osteoarthritis by Authors: Ellen M Gravallese, MD Ratnesh, Chopra MD https://www.ncbi.nlm.nih. gov/pmc/articles/PMC1753054/\#. No single histologic feature or group of features in synovium is diagnostic of rheumatoid arthritis (RA). Many of the histologic changes that are seen can also occur in other inflammatory joint diseases and even in osteoarthritis. Thus, the diagnosis of RA is made by history and physical examination of the patient and is supported by the presence of characteristic pathologic findings in synovial tissue, this conclusion raise the question of how many indefinite incorrect diagnoses based on histopathology of synovial biopsy alone.

Rheumatoid arthritis is a chronic disorder for which there is no known cure. Fortunately in the last few years, a shift in strategy toward the earlier institution of disease modifying drugs and the availability of new classes of medications have significant improvements in RA symptoms, slowed disease progression, and improved physical function and quality of life for patients. But
Swedish researchers have found no significant difference among death rates for those treated with 3 biological drugs" Larry Hand August 10, 2012" and another research "Rheumatoid Arthritis Mortality Has Not Changed in 20 Years Janis C. Kelly February 18, 2014."

Tendon sheaths are lined by paratenon and synovium; therefore, the tendons are affected by the same disease process as joints. In fact, symptoms of tenosynovitis may occur before those of intra-articular disease. The affected sites are

1. The dorsal and volar aspects of the wrist, because the tendons are covered by synovium as they pass under the flexor and extensor retinaculum and under the wrist,

2. The volar aspect of the digits, because the tendons are covered by synovium in the fibro-osseous canals in the finger.

Synovitis of the tendons can cause pain, dysfunction, and eventual rupture of tendons.

Surgical treatment in rheumatoid arthritis is used to relieve severe pain and improve function of severely deformed joints that do not respond to medication and physical therapy.

Synovectomies: removal of the diseased synovium is the most effective local treatment of RA, done in early stage in the hope to stop the process of destruction and prevent the invasion of tissues covered by synovium. 
There are two types of synovectomies:

1. around tendons, either flexor or extensor tenosnovectomy

2. around joint, synovectomy of the joints

Arthroplasty and partial or complete arthrodesis. In advanced stage synovectomy should be combined with stabilization procedures and correction of the deformity. Again the management of this generalized disease is primarily a medical problem.

\section{Case 1}

51 years old Sudanese lady suffering from long standing Rheumatoid arthritis under treatment with disease modifying drugs including biological drugs under care of Rheumatology team in another facility for 5 years.

Referred to me as she is complaining of painful swelling of the volar aspect of her right hand insidious onset progressive course of 6 months duration with no relief, she noticed also her little finger and index finger cannot be flexed with weak flexion of other fingers.

On examination there was sever flexor tenosynovitis with rupture both flexor tendons of little finger and index fingers and weak flexion of other fingers associated with painful swelling of the wrist joint extending distally to the palm and proximally to the forearm. (Figure1) Via volar approach( Figure $2 \& 3$ ) and after isolation of the median nerve and its superficial palmar branch both were stuck and adherent to the flexor retinaculum(Figure 4) which was opened, to me it was a surprise to find a large cold abscess with underlying rice bodies (Figure $5 \& 6$ ) sever tenosynovitis, rupture of the flexor tendon of the little and index fingers, a meticulous debridement, flexor tenosynovectomies, rerouting of one of the flexor superficial to regain movement power for the index and little finger (Figure 7-10) sample of the pus as well soft tissues and synovial tissues (Figure 11) sent both for culture and histopathology which turn to be and confirm the diagnosis of tuberculous flexor tenosynovitis, post-operative passed uneventful after receiving anti tuberculous drugs by infectious disease specialist.
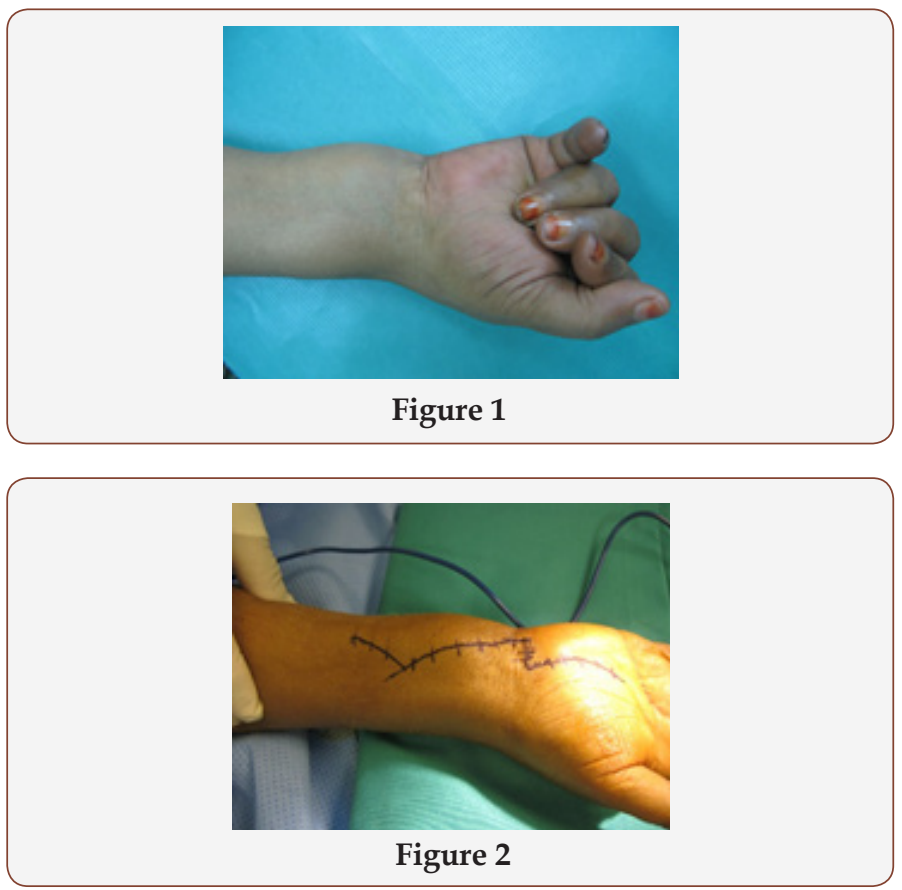
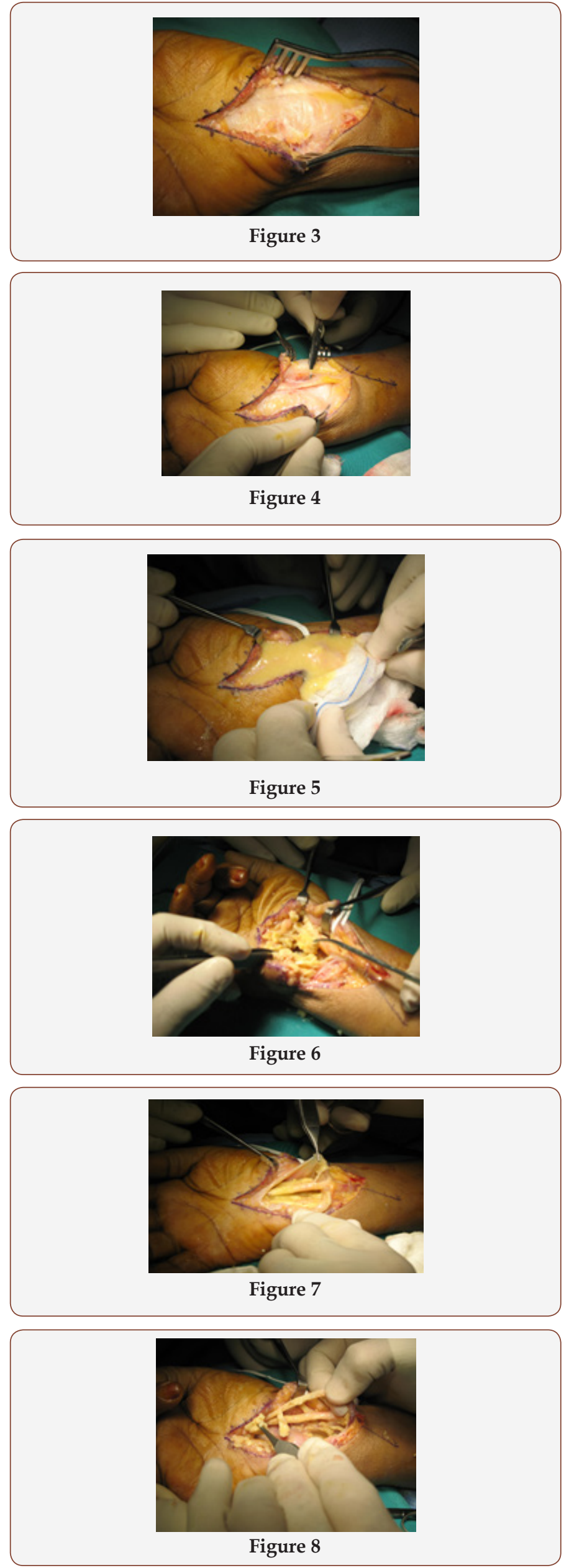


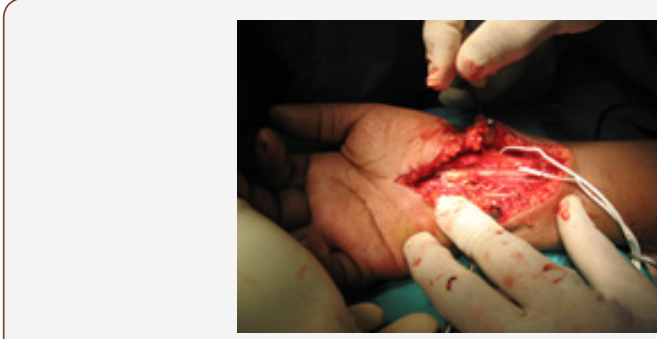

Figure 9

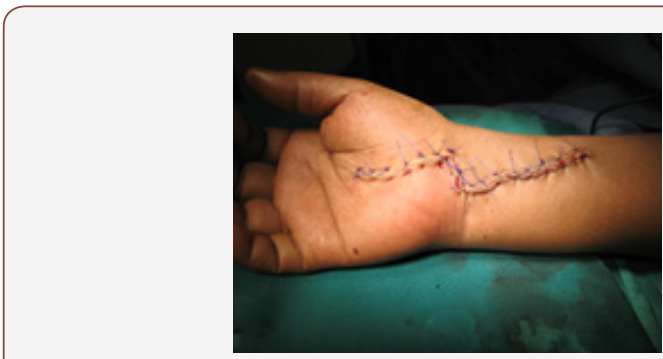

Figure 10

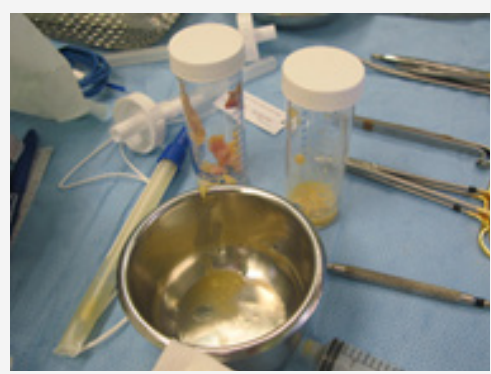

Figure 11

\section{Case 2}

35 years old Egyptian patient hand worker referred to me from the Rheumatology department in another facility as a case of rheumatoid arthritis with sever painful swelling due to flexor and extensor tenosynovitis right wrist insidious onset progressive course of few months duration with no history of other chronic illness or trauma, patient received treatment as a case of rheumatoid arthritis with inflammatory tenosynovitis with no relief, was referred to me because of intolerable pain and diminished R.O.M.

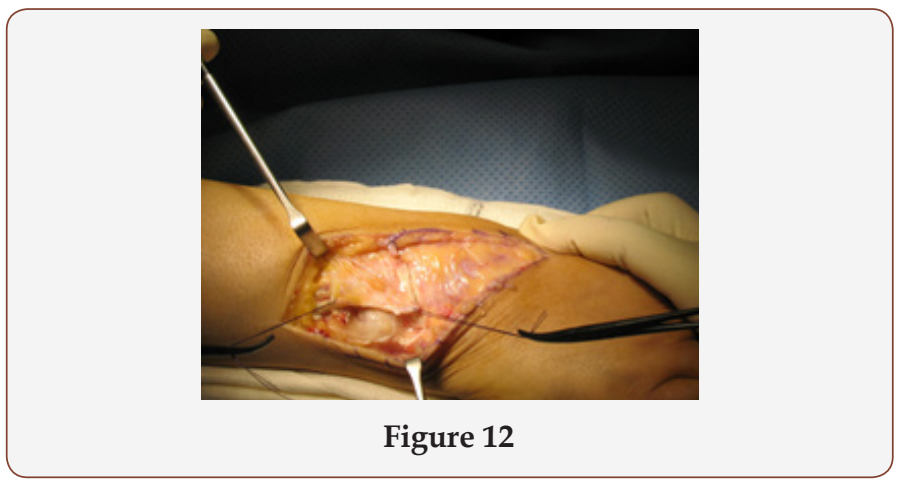

Via dorsal oblique and reflection of the extensor retinaculum (Figure 12 \& 13) the diseased synovium were excised both in extensor tendons (Figure 14) and the wrist joint (Figure 15) with a lot of erosions affecting the joint cartilage (Figure 16 \& 17) then another flexor tenoysnovectomy was done through volar approach
(Figure 18). All synovia removed sent for both histopathology examination then I found it is reasonable to send it for culture after the first experience, the result confirm surprisingly the diagnosis of TB tenosynovitis. Post-operative passed uneventful with patient improved well after receiving anti tuberculous drugs under proper care.
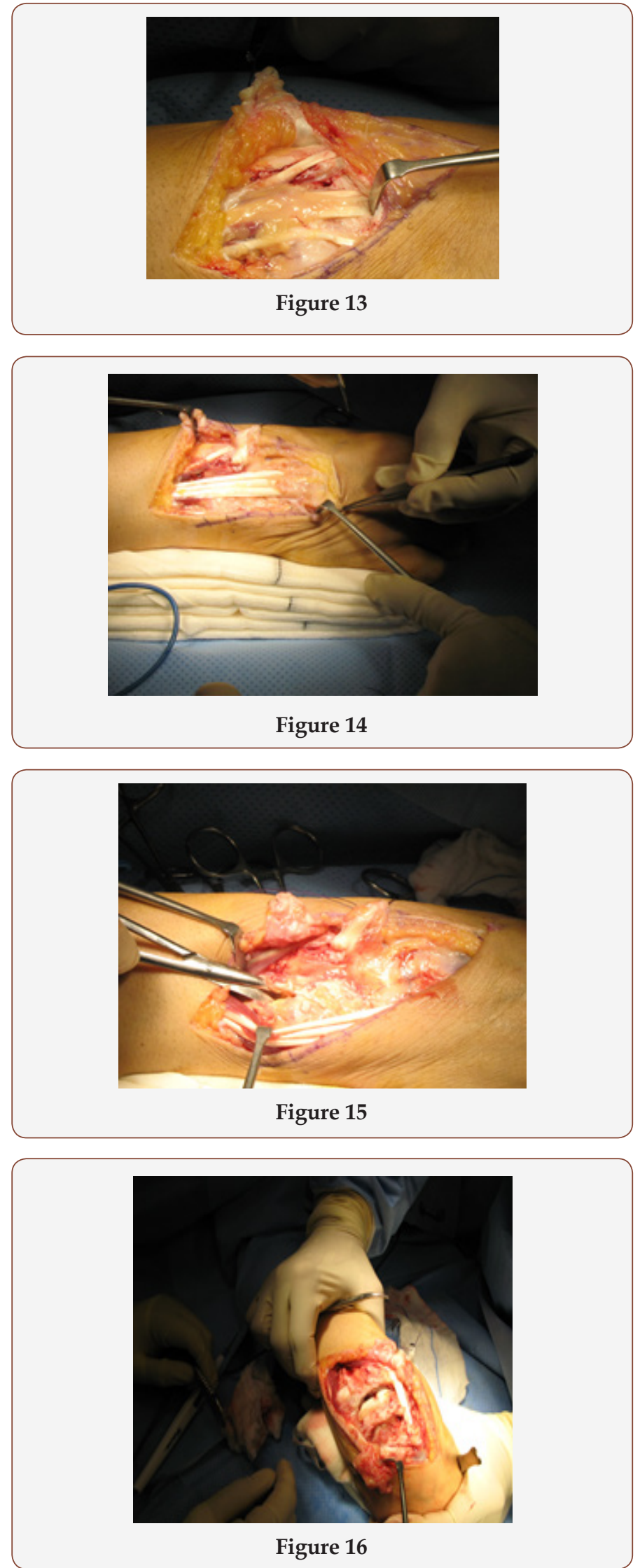

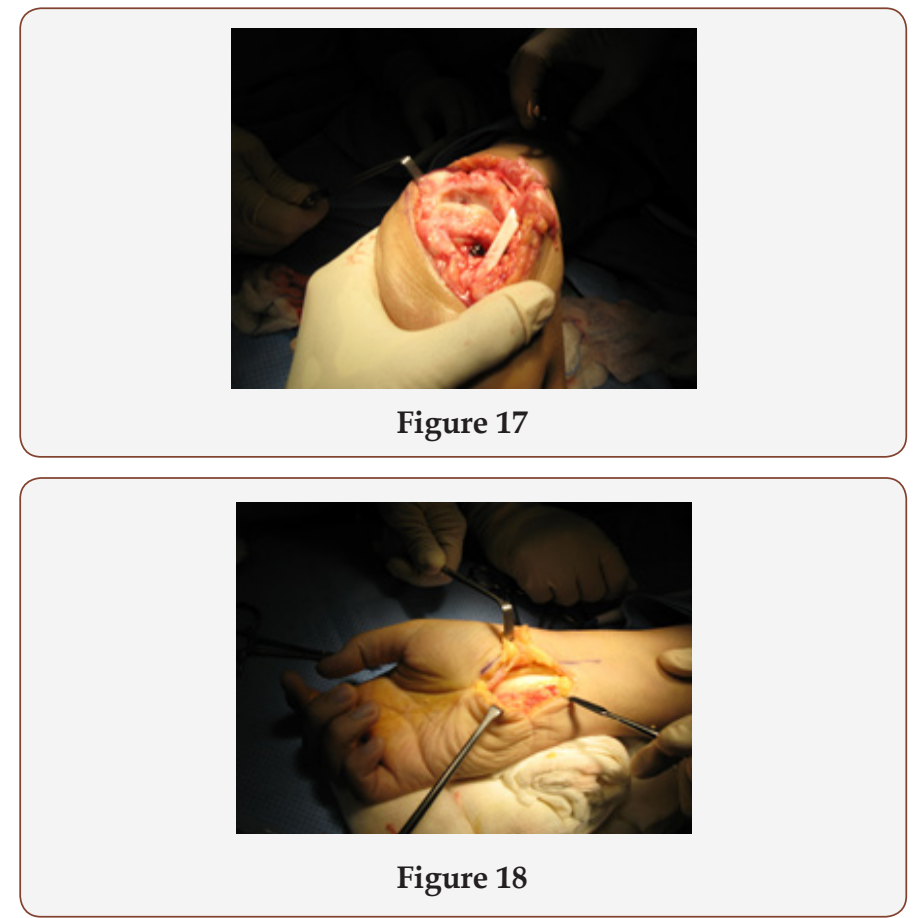

Case 3

33 years old Yemeni young chap suffering of intolerable painful swelling with diminished R.O.M in his left wrist for the last 6 months, he attended few clinics and was diagnosed as rheumatoid arthritis with sever tenosynovitis and he was receiving treatment including biological drugs with some relief from time to time.
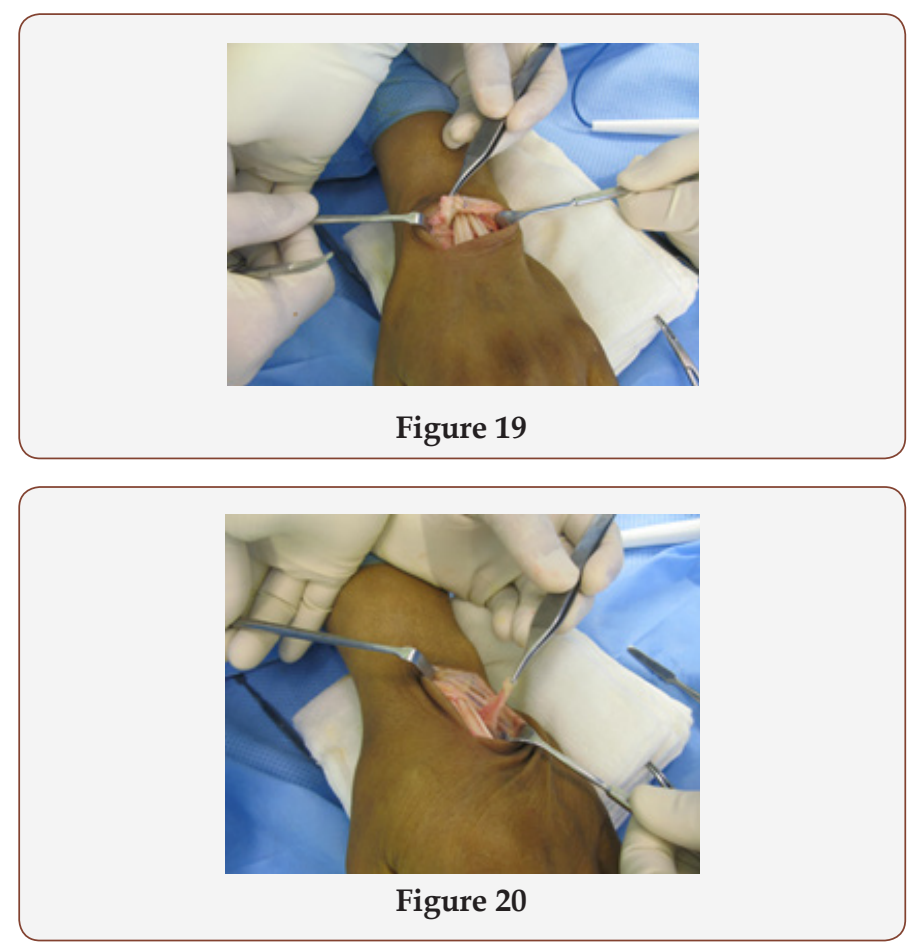

Patient refer to me from rheumatology clinic because of intolerable pain, for synovectomy and biopsy as a last resort. Via dorsal approach extensor (Figure 19), tenosynovectomy was done (Figure 20) and the synovium excised was sent for histopathology and for culture, again it was a surprise to me that it turn to be TB tenosynovitis. Post-operative passed uneventful and patient received a course of anti tuberculous with a lot of improvement

\section{Discussion}

Mycobacterium tuberculosis remains a top-10 cause of death worldwide, with greater than 2 billion active cases occurring mostly in developing countries. Tuberculosis is spread primarily by inhalation of infected Droplets, resulting in pulmonary disease, although rare cases of gastrointestinal and transdermal spread have been reported. Approximately $1 \%$ to $3 \%$ of patients infected with tuberculosis demonstrate skeletal involvement, most often manifested as spondylitis, peripheral arthritis, or osteomyelitis. Approximately $50 \%$ of patients with musculoskeletal tuberculosis have concomitant pulmonary disease. Tuberculous tenosynovitis is a rare complication of the disease, the onset of tuberculous tenosynovitis is gradual, with progressive swelling, pain, and diminished range of motion. Tendon sheaths of the hand and wrist are most frequently involved. The disease takes on three stages as it progresses: the earliest hygromatous form, a serofibrinous form, and a fungoid form, with considerable overlap of the three stages at presentation.

\section{Stages}

1. The hygromatous form usually appears as a serous exudates within a normal-appearing tendon sheath (Figure 19 \& 20).

2. The serofibrinous form (Figure 12-18) is manifested by obliteration of the tendon sheath with fibrous tissue and caseous inflammatory debris. Rice bodies appear in the synovial fluid, and involvement of the tendon itself with granulation tissue is seen.

3. Intertendinous adhesions may form, or complete rupture may occur.

The fungoid stage (Figure 2-11) involves extensive caseation and granulation tissue formation, causing obliteration of the tendon and sheath with formation of sinus tracts and a cold abscess. The three cases which I met in my career represent the 3 different stages of tuberculous tenosynovitis as you can see in the figures, I started to search about the link. Approximately 5\% of all individuals infected with Mycobacterium tuberculosis will develop active tuberculosis within the first two years after infection. Latent tuberculosis infection is defined as the period between the moment at which the bacilli enter the organism and the onset of active tuberculosis. The remaining $95 \%$ of exposed individuals will prevent the development of the disease via an effective cellular immune response. Elements that can affect immunity, such as coinfection (e.g., with HIV), comorbidity (e.g., diabetes mellitus), age, use of immunosuppressant's and nutritional status, promote the development of the disease. Rheumatoid arthritis (RA) is a comorbidity that has gained considerable interest in the last decade. There were a lot of studies and researches connecting rheumatoid arthritis treatment especially biological drugs and increases the incidence of TB

Pfizer research scientist Jim Mobley finds surprising links between arthritis and tuberculosis (, published Sunday, March 2, 2014.) First, he saw throughout scientific studies that animals 
injected with one species of bacteria were most likely to contract RA. This species was Mycobacterium tuberculosis. Second, he found interesting cases involving Enbrel, a drug used to treat RA. Enbrel blocks tumor necrosis factor, a chemical messenger that alerts surrounding cells of infection. While blocking TNF stops an overactive immune system from attacking itself, it also sometimes caused people who carried the tuberculosis bacteria to contract TB. This may be because TNF is important for TB immunity. The higher incidences of RA "almost perfectly mirrored" the death rates from TB, Mobley said. A certain Native American population had peak death rates from TB nearly seven times higher than those in England and North America over the last two hundred years. Generations later, this same population had rates of RA nearly seven times higher than the rest of the world. In Africa, where TB had been relatively nonexistent, RA was now also absent "Mobley published his idea in the journal Medical Hypotheses, asking "Is rheumatoid arthritis a consequence of natural selection for enhanced tuberculosis resistance?"”

Rothschild found that prior to 1785 there was no record of RA in Europe - only after the occurrence of TB epidemics. This suggested the epidemics were responsible for the existence of RA." "Genetic mutations that allowed people to survive tuberculosis epidemics two hundred years ago are combining and coming together in the descendants of those individuals, making their immune systems very much stronger and unfortunately, inducing these autoimmune-type diseases," he said. Data from the British Society for Rheumatology Biologics Register (BSRBR) Oct 22, 2009, a national prospective observational study, were used to compare TB rates in 10712 anti-TNF treated patients, Conclusion The rate of TB in patients with RA treated with anti-TNF therapy was three- to fourfold higher in patients receiving INF.

Patients receiving biologics are at higher risk for developing tuberculosis. New cases of tuberculosis or reactivation of latent tuberculosis infections may occur during the course of treatment," Rheumatic Disease Clinics of North America Volume 35, Issue 1, Pages 163-181, February 2009". We found that the use of biological and traditional DMARDs is associated with an increased risk of developing TB in patients with RA, mainly among noncurrent users of corticosteroids. Clinical Infectious Diseases, Volume 43, Issue 6, 15 September 2006, Pages 717-722, https://doi. org/10.1086/506935.

A study shows that tuberculosis infection (TB) is 10 times more common among people who have rheumatoid arthritis than it is in the general population, and that risk appears to be elevated by treatment with any immunosuppressive medication, not just biologic drugs." 03/20/2009 | By Jennifer Davis", But the new study, by researchers at McGill University Health Center in Montreal, Quebec, Canada, suggests that the risk is increased for anyone with rheumatoid arthritis who is taking a corticosteroid such as prednisone, or a disease modifying anti-rheumatic drug (DMARD), such as methotrexate (Trexall, Rheumatrex) or leflunomide (Arava).

Reactivation of latent tuberculosis infection (LTBI) has emerged as a problem in patients with rheumatoid arthritis (RA). Anti-tumor necrosis factor $\alpha$ therapy is strongly associated with reactivation of tuberculosis (TB) in patients with RA. However, RA itself has been associated with a higher incidence of TB. The current consensus is that patients with RA should be screened and, if necessary, treated for LTBI before the start of treatment for RA and monitored closely with yearly tests for TB. There is no gold standard for making the diagnosis." Latent Tuberculosis Infection in RA: The Disease and the Diagnosis June 27, 2011" http://www.rheumatologynetwork.com/ rheumatoid-arthritis.

Tuberculosis Linked to Arthritis Drugs Doctors Should Screen for TB before Prescribing Drugs That Suppress Immune System, 2004 WebMD, In, By Jeanie Lerche Davis. In special article "Tuberculosis in rheumatoid arthritis patients: the difficulty in making the diagnosis of latent infection" Since the beginning of the use of anti-TNF in the treatment of rheumatoid arthritis and other inflammatory diseases, cases of pulmonary tuberculosis and extra pulmonary tuberculosis have been reported in patients receiving such treatment. In most cases, the disease develops by the time the patient has received the sixth infusion. Every patient should be evaluated for latent tuberculosis infection prior to the use of a TNF inhibitor. However, the diagnosis of latent tuberculosis infection is a challenge. The tuberculin test, which was the only test available to detect latent tuberculosis infection for nearly a century, presents a number of limitations. Tests based on the detection of the in-vitro production of IFN- $\gamma$ by mononuclear cells activated by specific antigens appear to be more accurate and have been studied in patients with rheumatoid arthritis. http://www.scielo. br/scielo.php?pid=S1806-37132010000200014\&script $=$ sci $_{-}$ arttext\&tlng=en\#nota2.

IN a study published BY EGYPTIAN JOURNAL OF CHEST DISEASE AND TUBERCULOSIS “January 2015, Pages 109-113” conducted on (235) RA patients indicated for either conventional therapy or antiTNF therapy from 1-1-2010 to 1-10-2013., There was no significant increased risk for tuberculosis among RA patients receiving antiTNF therapy when screening and chemoprophylaxis was applied, so screening of RA patients before anti-TNF therapy for latent tuberculosis and TB chemoprophylaxis should be done. https:// www.sciencedirect.com/science/journal/04227638/64/1.

\section{Our Lessons}

1. 1.Always send a biopsy for culture and sensitivity after excision of any synovial sheath, necrotic tendon or soft tissues.

2. T.B. tenosynovitis of extensor and flexor tendons and synovitis of the wrist joints became strongly one of the D.D. of chronic arthritis of the wrist even in a proven cases of rheumatoid arthritis.

3. Prompt surgical debridement and tissue diagnosis are essential for the diagnosis and treatment of this type of infection. With an accurate and timely diagnosis, appropriate surgical and anti-tuberculous treatment may eradicate these unusual infections.

\section{Conclusion}

- Always screen before treatment

- $\quad$ Always culture 


\section{References}

1. MF Doran, CS Crowson, GR Pond, et al. (2002) Frequency of infection in patients with rheumatoid arthritis compared with controls: a population-based study. Arthritis Rheum 46(9): 2287-2293.

2. F Wolfe, K Michaud, J Anderson, K Urbansky (2004) Tuberculosis infection in patients with rheumatoid arthritis and the effect of infliximab therapy. Arthritis Rheum 50(2): 372-379.

3. Cantini F, Goletti D (2014) Biologics and tuberculosis risk: the rise and fall of an old disease and its new resurgence. J Rheumatol Suppl 91: 1-3.

4. Carmona L, Hernandez Garcia C, Vadillo C, Pato E, Balsa A, et al. (2003) Increased risk of tuberculosis in patients with rheumatoid arthritis. J Rheumatol 30(7): 1436-1439.

5. Bouza E, Moya JG, Munoz P (2001) Infections in systemic lupus erythematosus and rheumatoid arthritis. Infect Dis Clin North Am 15(2): $335-361$.
6. (2011) Latent Tuberculosis Infection: A Guide for Primary Health Care Providers. Diagnosis of Latent TB Infection. Centers for Disease Control and Prevention.

7. British Thoracic Society Standards of Care Committee (2005) BTS recommendations for assessing risk and for managing Mycobacterium tuberculosis infection and disease in patients due to start anti-TNFalpha treatment. Thorax 60(10): 800-805.

8. Mayordomo L, Marenco JL, Gomez Mateos J, Rejon E (2002) Pulmonary miliary tuberculosis in a patient with anti-TNF-alpha treatment. Scand J Rheumatol 31(1): 44- 45.

9. Elbek O, Uyar M, Aydin N, Börekçi S, Bayram N, et al. (2009) Increased risk of tuberculosis in patients treated with antitumor necrosis factor alpha. Clin Rheumatol 28(4): 421- 426.

10. Dixon WG, Hyrich KL, Watson KD, Lunt M, Galloway J, et al. (2010) Drugspecific risk of tuberculosis in patients with rheumatoid arthritis treated with anti-TNF therapy: results from the British Society for Rheumatology Biologics Register (BSRBR). Ann Rheum Dis 69(3): 522- 528. 